\title{
Image-based, unsupervised estimation of fish size from commercial landings using deep learning
}

\author{
Amaya Álvarez-Ellacuría, Ignacio A. Catalán, Miquel Palmer \\ IMEDEA(CSIC-UIB), Spain \\ amaya@imedea.uib-csic.es \\ Jose-Luis Lisani \\ Universitat de les Illes Balears, Spain \\ joseluis.lisani@uib.es
}

\begin{abstract}
The dynamics of fish length distribution is a key input for understanding fish population dynamics and to take informed management decisions on exploited stocks. Nevertheless, in almost all fisheries, estimating length of landed fish is still made by hand. As a result, length estimation is precise at fish level but, due to the inherent high costs of manual sampling, sample size tends to be small. Accordingly, precision of population level estimates are often suboptimal and even prone to bias when properly stratified sampling programs are not affordable. Conversely, recent applications of machine learning and artificial intelligence to fisheries science are opening a promising opportunity for massive, unbiased sampling of fish catches. Here we present the results obtained using a deep convolutional network (Mask R-CNN) for unsupervised (i.e., fully automatic) fish length estimation from images of fish boxes obtained at the auction center. In the case of European hake, the network accurately identifies and segments heads of fish with a percentage of false positives close to $2.2 \%$. Concerning accuracy and precision of fish length estimates, the 95th quantile of the error (observedestimated) ranged from -5.9 to $4.5 \mathrm{~cm}$, with a median value very close to zero.
\end{abstract}

Keywords: fish size estimation; landings; deep learning; convolutional neural networks 


\section{Introduction}

Ensuring fish stocks sustainability while maximizing fishers profitability is an elusive and still not solved topic (Hilborn, 2007; Iudicello et al., 2012). Solving this puzzle is specially urgent in the case of the Mediterranean fleets because they are going through a deep crisis for decades, which has been attributed to the continuous decrease in the sale price of fish, which translates in a continuous decrease in the number of boats and in a very low recruitment rate of young fishermen (Palmer et al., 2017). At least in the case of the Balearic Islands, the root of the problem seems to be more related with the commercialization of the product that with the state of conservation of the stocks (Morales-Nin et al., 2010; Maynou et al., 2013; Reglero and Morales-Nin, 2008).

Co-management (d'Armengol et al., 2018) is one of the strategies aimed to match stocks sustainability and fisher profits. Several small-scale fisheries are currently co-managed in the Balearic Islands (e.g., Aphia minuta and Coryphaena hippurus) suggesting that fishers are prone to adopt this type of strategy. Co-management delivers both ecological and social benefits (d'Armengol et al., 2018) but periodical, adaptive review of the results are mandatory in order to adopt short-term, operational management decisions. However, those decisions must be informed by accurate and precise data. Similarly, conventional fishery models may inform on the mid- and long-term trends of the exploited stocks but must be feed with accurate and precise data too.

One of the key variables needed for both taking short-term management decisions and modeling stock trends is fish length. Nevertheless, in almost all fisheries, estimating the length of landed fish is still made by hand. Length measures are precise enough for those purposes, but provided that observers' cost is high, the sample size used for estimating length at population level tends to be relatively small. Accordingly, the estimation of average length at its spread at the population may be imprecise and may be prone to bias when properly stratified sampling programs are not affordable.

In contrast with the relatively low efficiency of observers, a massive amount of images can be processed by computer vision. Some of the earliest attempts to use computer vision techniques for length measurement of fish were reported by Arnarson (Arnarson et al., 1991) and Strachan (Strachan, 1993). In both cases a camera was placed on top of a conveyor belt where fishes passed by, one at a time. The illumination conditions were controlled in such a way that fishes were much darker than the background. Therefore, a simple illumination threshold was used to detect them. Once detected, their orientation was determined and normalized and the (possibly curved) line from nose to tail fork was computed. The length of this line was used to estimate the actual length of the fish. More complex versions included edge detection, color calibration and the distinction between roundfish and flatfish (White et al., 2006). However, the system setting (conveyor belt with controlled lighting) was similar. In a similar way too, pictures of individual fishes are used as inputs of the system and edge and corner detection methods are applied to estimate the position of head and tail from which the length is computed (Abdullah et al., 
2009).

Detection and measurement of live fish in underwater images is more challenging. Concerning detection, body silhouettes have been extracted using edge detection techniques under controlled illumination conditions (Hardin, 2006; Zion et al., 2007; Miranda and Romero, 2017). Stereo methods and 3D models have been proposed to concurrently estimate the fish length and the distance of the free-swimming fish from the camera (Petrell et al., 1997; Tillett et al., 2000; Díaz-Gil et al., 2017). Image enhancement techniques for the correction of color and illumination have been implemented too (Martinez-de Dios et al., 2003; Costa et al., 2006; Al-Jubouri et al., 2017). A common characteristic of these methods is that, once distance to camera and illumination have been normalized, they use classical image processing techniques (segmentation by thresholding or edge/corner detection) to extract the fish features of interest.

However, those conventional image processing techniques have been progressively replaced by methods based on machine learning for the tasks of detection and classification. One of the first applications was the detection of human faces (Viola and Jones, 2004). Subsequently, techniques based on Support Vectors Machines (SVM) and the use of SIFT and HOG descriptors (Dalal and Triggs, 2005) obtained notable results in the detection of various types of objects (faces, vehicles, pedestrians, etc.). The use of convolutional neural networks $(\mathrm{CNN})$ as a pattern recognition model based on biology received a strong boost in 1990 with the use of the backpropagation technique for network training LeCun90 (Lecun et al., 1998). However, the techniques based on CNNs fell into disuse until in 2012 when classification results of images far superior to the state of art at that time were reported thanks to the use of a deep network (with many layers) and a large amount of data for training (Krizhevsky et al., 2012), giving rise to the current boom of deep learning. Since 2012, increasingly deep networks have been proposed and have been applied to problems as diverse as detection, classification, segmentation, etc. and for different types of images (vehicles, pedestrians, faces, etc). Some of the most popular models for detection are YOLO (Redmon et al., 2016) and Mask R-CNN (He et al., 2017).

In the case of fish detection, the use of deep learning techniques is incipient and faces the additional problem that fish are not rigid objects and networks must learn how to adapt to changes in posture, position and scale. Nevertheless, fish recognition has been achieved using a binary classifier (Marini et al., 2018) or a neural network with only two convolutional layers and a linear SVM classifier (Qin et al., 2016). In addition, existing network architectures (e.g., LeNet, AlexNet, GoogLeNet or YOLO) have been used for fish classification (Meng et al., 2018; Chen et al., 2017).

Hake (Merlucius merlucius) is considered overfished in the Mediterranean, with an alarming $20 \%$ reduction in catches in the last 20 years. Overfishing in the Balearic islands is moderate (FAO, 2016), but the overall status in the Mediterranean is critical (FAO, 2018). Moreover, this species represent an economically relevant fraction of the landings in the Balearic Islands (Palmer et al., 2009, 2017). 
Accordingly, here we propose the hake as case study species for adapting an existing network architecture (Mask R-CNN (He et al., 2017)) for massively estimating fish length from images. This strategy is technically feasible because images of fish boxes are routinely obtained at the conveyor belt, just at the bidding, in the auction center. Therefore, in the near future length estimates for all the fish boxes sold in a day, all the days of the year can be obtained at affordable cost, thus fully fulfilling the data requirements that would enable taking informed operational decisions at the short term scale needed for co-management scale and, at the same time, monitoring the mid- and long-term trends of the stock.

\section{Material and Methods}

\subsection{Sampling Images}

More than 500 images of hake boxes were obtained at the conveyor belt in the auction center of Palma (Mallorca, Balearic Islands) during 2018 using a webcam with a pixel resolution of 1280x920. The camera was placed top-down, just over the fish boxes and takes an image just at the bidding moment, when the conveyor belt stops for a while. For most of the species landed, including hake, fish are sorted by species by fishermen. Therefore, in those cases, there is no need for a preliminary classification task. Some examples of the images obtained are displayed in Figure 1.
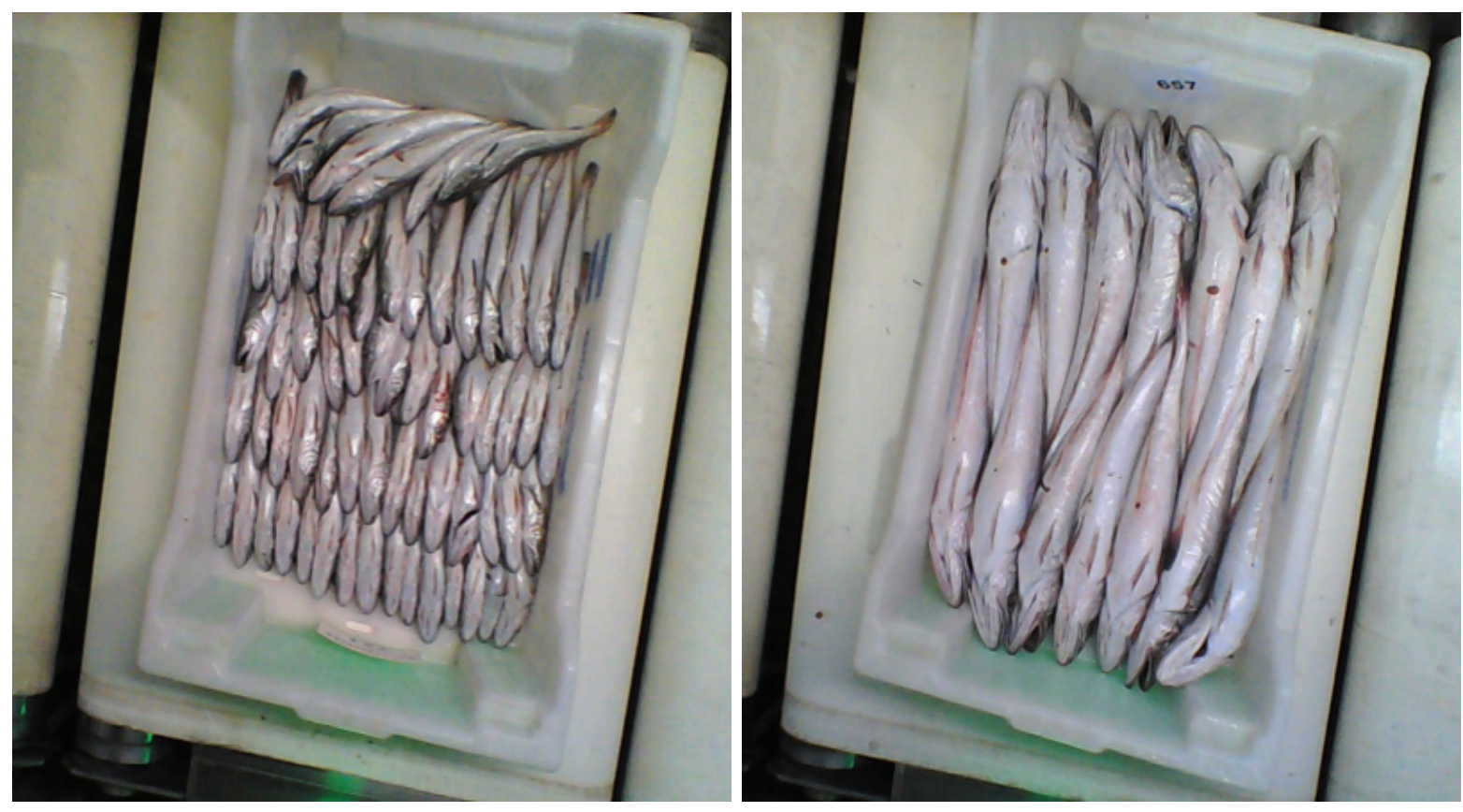

Figure 1: Input images. 


\subsection{Network Implementation}

The network used (Mask R-CNN (He et al., 2017)) is a simple, flexible, and general purpose network for object instance segmentation, which implies not only recognizing all the objects (or instances) from the target category in a given image but also accurately segmenting them. Mask R-CNN is based on Faster R-CNN (Ren et al., 2015), which focuses in object detection (i.e., each target object is enclosed into a rectangular bounding box), with an extension for creating a segmentation mask of the target object within the bounding box (Figure 2). Therefore, Mask R-CNN consists of two convolutional neural networks that work in parallel. A backbone architecture for the extraction of features over the entire image plus a head architecture for recognizing Regions of Interest (ROIs) and producing a mask over them. A scheme of the network architecture is displayed in Figure 3. The developers of Mask RCNN have shown that the proposed architecture outperforms more complex networks and that the best results are obtained with a ResNet-FPN (Lin et al., 2017) backbone with 101 layers and a fully convolutional network (FCN) head consisting of 6 convolutional layers.

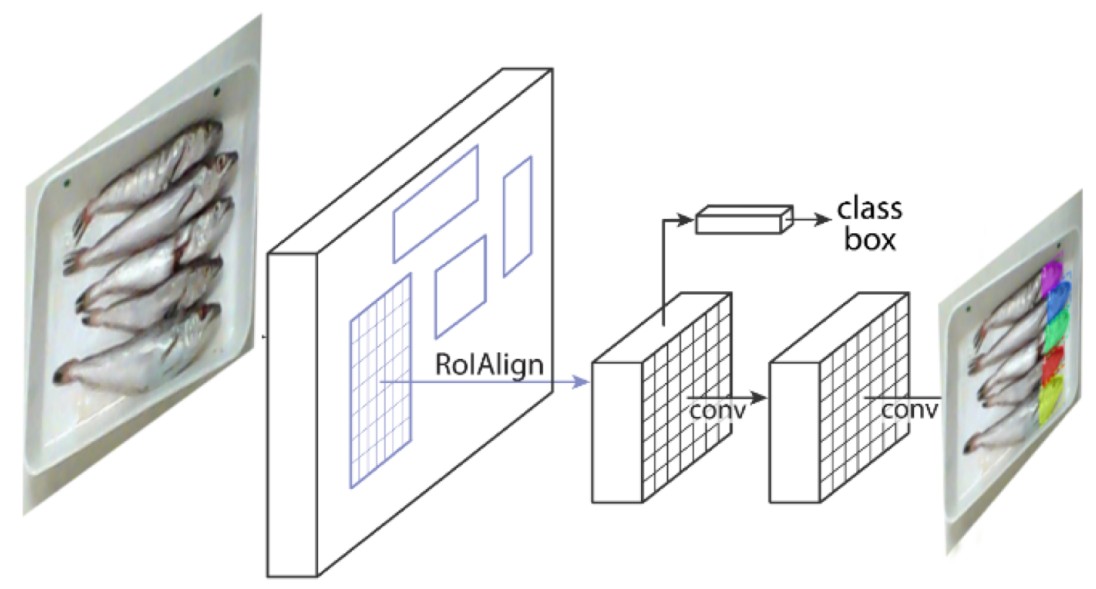

Figure 2: MASK-RCNN framework (modified from He et al. (2017)).

The implementation of Mask R-CNN used is available at Github ${ }^{1}$. This implementation uses Python 3, Keras and TensorFlow. Moreover, the network uses pre-trained weights from the COCO dataset (a public dataset of images available at http://cocodataset.org/). However, these weights must be fine tuned for the case-specific target and using a user-defined dataset.

In the case study, hakes are arranged inside a fish box in a way that in most cases the tails are occluded and a complete view of a fish is available for a few fish only. Accordingly, in the case of hake, the target object to be segmented cannot be the whole fish but only a part. Fortunately, many complete heads are visible on the images, thus fish heads have been the target object with which the network has been finally trained. The head has been defined here as the area from mouth to pelvic fin (Figure 4). The network was fine-tuned using more than 2,000 heads. Note that the contour of the fish heads to

\footnotetext{
${ }^{1}$ https://github.com/matterport/Mask_RCNN
} 


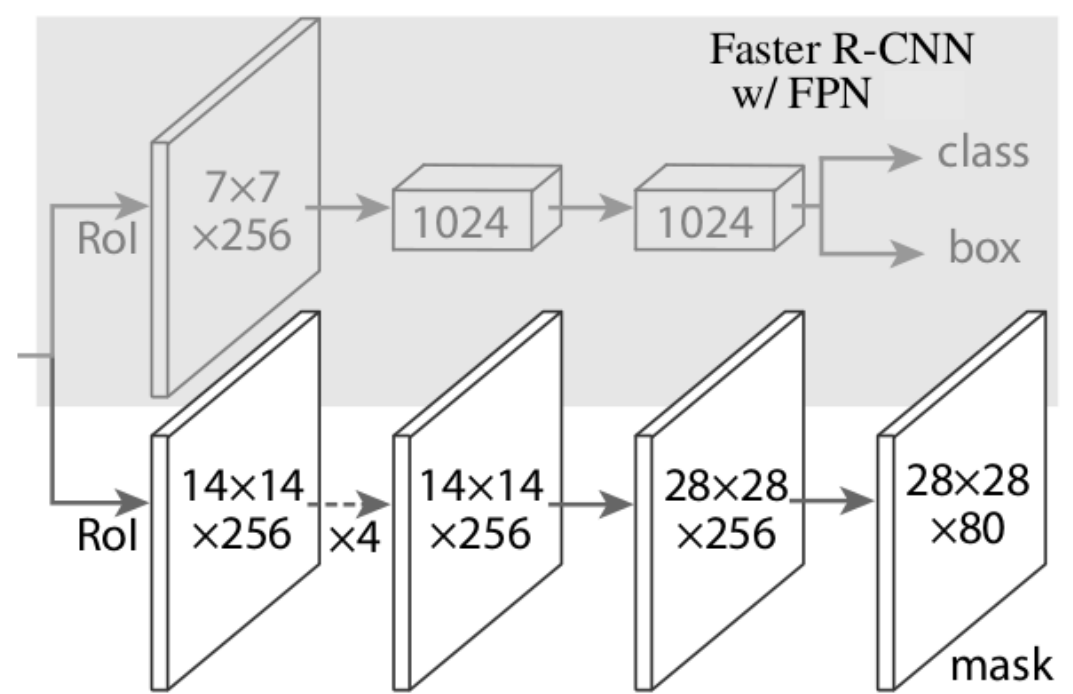

Figure 3: MASK-RCNN Architecture from He et al. (2017).

be used for training the network has been manually segmented. Concerning the training process, the setting was 100 epochs, with 200 steps per epoch, and 50 validation steps. The learning rate was 0.002 and only weights of the head branch of the network were learned using the training set.

\section{Total length}

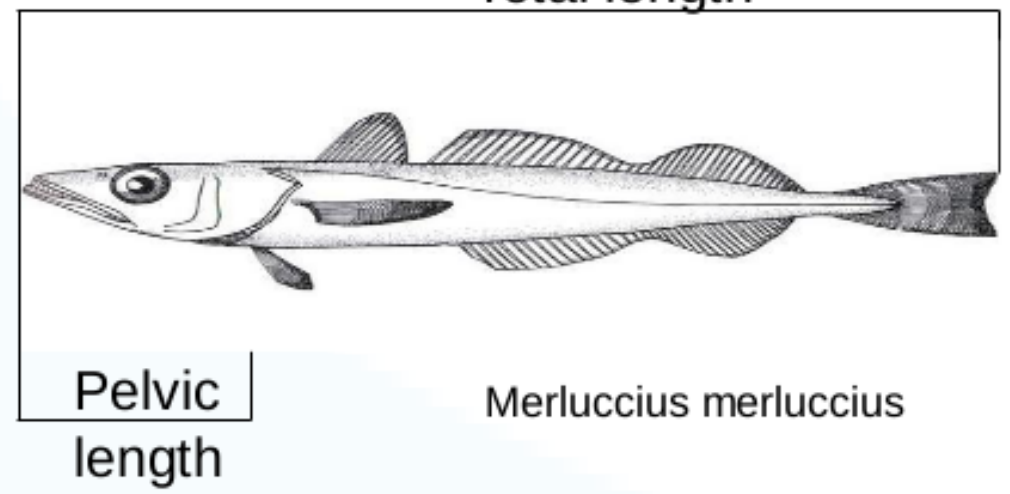

Figure 4: Head definition: from mouth to pelvic fin.

\subsection{Network Validation}

After training, the network was used to identify heads on new images that have never been used for training. The performance of the network was assessed in two ways. First, from the percentage of false positives (i.e., objects that have been wrongly identified as fish heads). Note that in this specific context, the false negatives (heads that has not been identified) are irrelevant because it is not necessary to measure all the fish in a box for getting an accurate estimate of the fish distribution in that box.

The second performance metric is the accuracy and the precision attained when estimating the fish 
length itself. The strategy for estimating the fish length comprises three steps: 1) unsupervised estimation of head length (HLpix, pixels) from an image using Deep Learning; 2) estimation of head length (HLcm, $\mathrm{cm}$ ) from HLpix; and 3) estimation of body length (TL, cm) from HLcm.

For estimating accuracy and precision of the method, total body length (TL, from the tip of the mouth to the tip of the tail) of a sample of hakes was measured once using a ruler with an instrumental precision of $1 \mathrm{~mm}$. Similarly, head length (HLcm, from the tip of the mouth to the insertion point of the ventral fin) of the same hakes was measured once using the same ruler. Finally, the images of those sampled fish was taken emulating the actual environmental conditions. Specifically, fish were visually labeled with an ID and were placed on fish boxes in groups of 5-10 fish. Several images from each group were taken after changing the posture of all fish in the box. Those images were submitted to the unsupervised routine for head segmentation described above.

The sampling protocol above was designed to estimate three main sources of variability. Namely, 1) variability between the repeated estimates of HLpix from the same fish (i.e., repeated measures of HLpix from the same fish from different images after changing fish posture), 2) variability related with the (imperfect) relationship between HLpix and HLcm, and 3) variability related with the (imperfect) relationship between HLcm and TL.

The statistical model assumed that the $j$ repeated measurement for HLpix of the same fish $i$ from different images after changing fish posture is normally distributed around a mean value HLpix, with a an standard deviation $\sigma_{\text {HLpix }}$ :

$$
\operatorname{HLpix}_{i, j} \sim N\left(\overline{\operatorname{HLpix}}_{i}, \sigma_{\text {HLpix }}\right)
$$

Concerning the relationship between HLpix and HLcm, a lineal model with zero intercept was considered:

$$
\begin{aligned}
\overline{\operatorname{HLpix}}_{i} & =\beta_{\mathrm{head}} \mathrm{HLcm}_{i}+\varepsilon_{\mathrm{HLcm}_{i}} \\
\varepsilon_{\mathrm{HLcm}_{i}} & \sim N\left(0, \sigma_{\mathrm{HLcm}}\right)
\end{aligned}
$$

were the overline indicates the expected mean, $\beta_{\text {head }}$ is the slope of the lineal relationship and $\varepsilon_{\mathrm{HLcm}}$ is a normally distributed error with zero mean and standard deviation $\sigma_{\mathrm{HLcm}}$. Concerning the relationship between HLcm and TL, four lineal models resulting for log-transforming or not these variables were compared. The model finally selected (see results below) was:

$$
\begin{aligned}
\mathrm{TL}_{i} & =\alpha_{\text {body }}+\beta_{\text {body }} \log \left(\operatorname{HLcm}_{i}\right)+\varepsilon_{\mathrm{TL}_{i}} \\
\varepsilon_{\mathrm{TL}_{i}} & \sim N\left(0, \sigma_{\mathrm{TL}}\right)
\end{aligned}
$$

were, $\alpha_{\text {body }}$ and $\beta_{\text {body }}$ are the intercept and the slope of the lineal relationship and $\varepsilon_{\mathrm{TL}}$ is a normally distributed error with zero mean and standard deviation $\sigma_{\mathrm{TL}}$. 
For the first submodel (1), 210 estimates of HLpix from 70 fish were considered (repeated measurements ranged from 2 to 5 per fish). For the second submodel (2), HLpix and HLcm from 60 fish were considered. Finally, for the third submodel (3), TL and HLcm from 98 fish were considered. Note that the three variables (TL, HLcm, HLpix) are all available for 60 fish.

The parameters of the integral model (i.e., combining the three submodels into a single analysis) have been estimated using a Bayesian approach implemented in a custom R scrip (https://www.r-project. org/) that runs JAGS (http://mcmc-jags.sourceforge.net/) for moving the MCMC chains. Three independent chains were run. The convergence of the MCMC chains was assessed by visual inspection of the chains and was tested using the Gelman-Rubin statistic (Plummer et al., 2006). A threshold value between 0.9 and 1.1 was assumed to suggest convergence (Gelman et al., 2003). Virtually flat priors were used: normal distribution with zero mean and a huge variance was assumed for $\beta_{\text {head }}, \alpha_{\text {body }}$ and $\beta_{\text {body }}$. Gamma distributions (rate $=0.01$, scale $=0.01$ ) were assumed for the three standard deviations $\left(\sigma_{\mathrm{HLpix}}, \sigma_{\mathrm{HLcm}}\right.$ and $\left.\sigma_{\mathrm{TL}}\right)$. Posterior distribution was estimated by at least 30.000 valid iterations after appropriate burning and thinning (one out 10 iteration were keep).

\section{Results}

The Mask R-CNN was successfully implemented according with the developers specifications and fine tuned with a training data set composed by more than 2,000 manually segmented heads.

Concerning the first validation metric, in the 68 photos used as input for the validation of the network, there were a total of 292 hake heads. Of these, $288(98.6 \%)$ were detected correctly, so the network presents an excellent efficiency in terms of false negatives (that is, not detecting a head when in fact there is a head). Concerning false positives (that is, retaining an object when it is not a head), 9 cases were detected, although one of them was an object outside the box and therefore an automatic quality control can be easily implemented to filter for those types of false positives. Considering only the false positives within the boxes, its ratio in relation to the number of successes is very low $(2.8 \%)$, as was the ratio between false positives and the true number of heads (2.7\%) Some examples of the output of the network are displayed at Figure 5. 

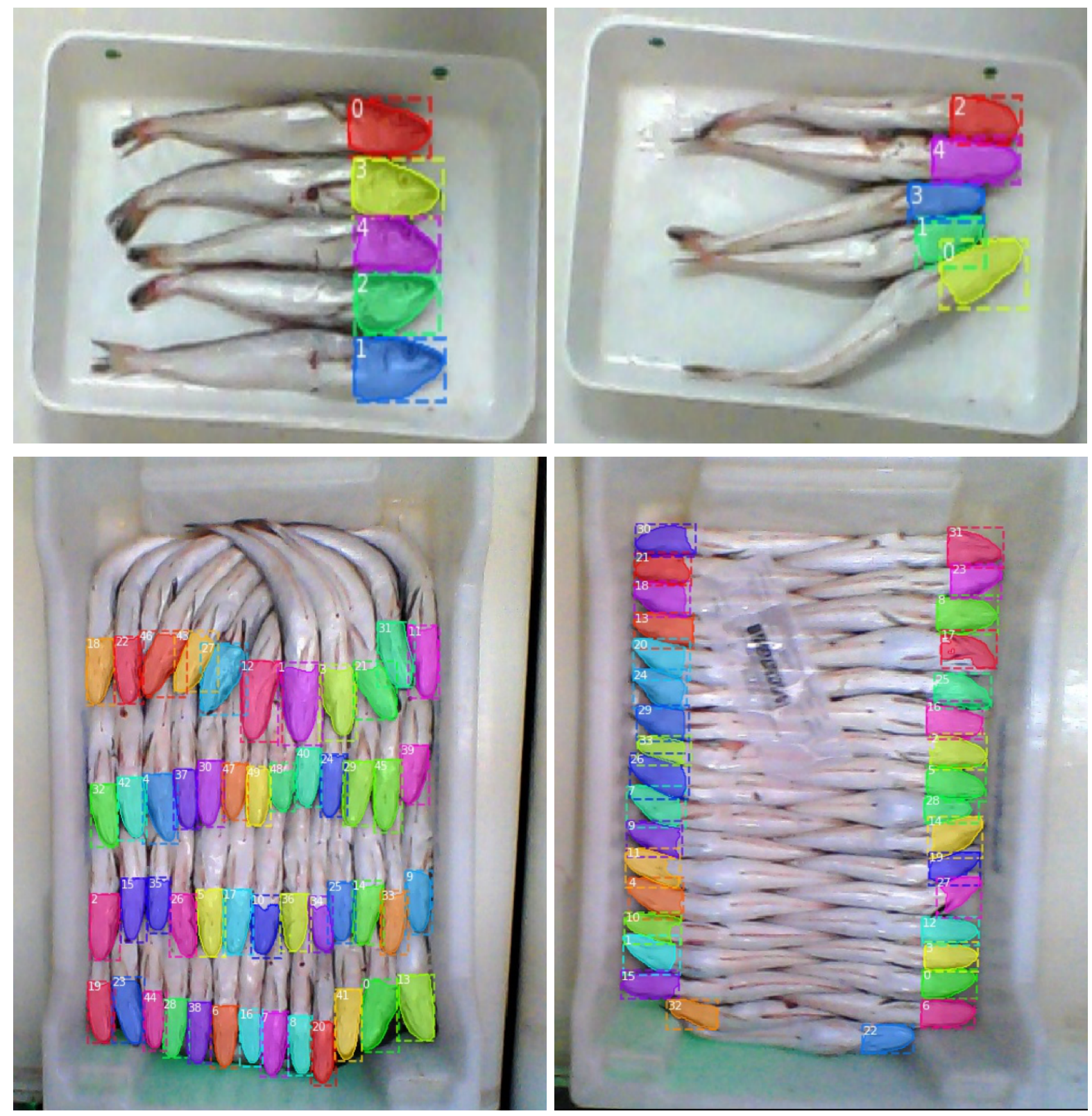

Figure 5: Some segmentation results.

Note that Mask R-CNN produces a score measuring the confidence in the fact that an actually retained object belongs to the category of interest. It is specially noteworthy that the few false positives have low confidence scores (Figure 6). Therefore, a post-processing routine can be easily implemented for filtering out most of the false positives based on the confidence score.

The second performance metric is the accuracy and the precision attained when estimating the fish length itself. Preliminary analyses of the relationship between HLcm and TL showed that the four lineal models, either using log-transformed values or not, had an excellent explanatory power, with adjusted r squared larger that 0.95 . However, residual distribution displayed clear biases in the cases of using non-transformed HLcm as explanatory variable. From the other two cases, residuals seem more evenly distributed in the case of TL versus $\log (\mathrm{HLcm})$, thus this model was finally selected. 


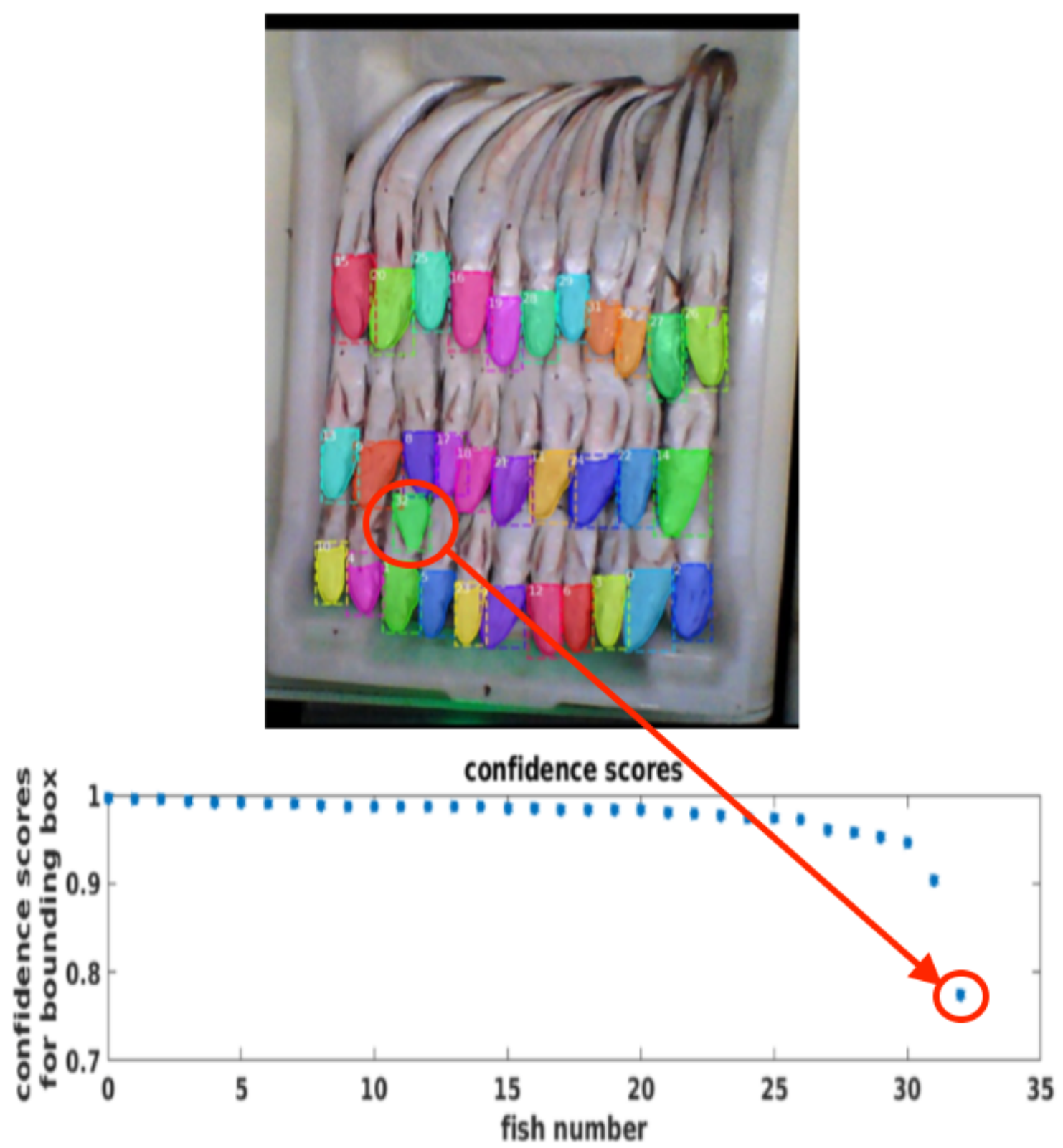

Figure 6: Top, picture containing a false detection. Bottom, the confidence value for the false detection is much smaller than for the rest of detections.

All parameters of the model have been successfully estimated given the data and MCMC chains have nicely converged (see Table 1).

For assessing accuracy (degree of closeness of estimates of a quantity to that quantity's true value), $\mathrm{TL}_{\text {true }}$ of 70 fish ranging from 19.3 to $27.5 \mathrm{~cm}$ (i.e., the true value has been actually measured) was compared with $\mathrm{TL}_{\mathrm{est}}$ estimated from HDpix. The 95th quantile of the error (difference between the measurement and the estimation) ranged from -5.9 to $4.5 \mathrm{~cm}$, with a median value very close to zero, which suggests that the system is accurate. However, precision (dispersion of predicted values for a given observed value) may be improved. 


\begin{tabular}{l|ccc} 
& $2.5 \%$ & median & $97.5 \%$ \\
\hline$\alpha_{\text {body }}$ & -30.761 & -28.921 & -27.192 \\
$\beta_{\text {body }}$ & 30.681 & 31.619 & 32.627 \\
$\beta_{\text {head }}$ & 0.842 & 0.861 & 0.881 \\
$\sigma_{\text {TL }}$ & 1.343 & 1.528 & 1.768 \\
$\sigma_{\text {HLcm }}$ & 0.024 & 0.081 & 0.214 \\
$\sigma_{\text {HLpix }}$ & 0.311 & 0.369 & 0.445
\end{tabular}

Table 1: Median and 95\% bayesian credibility interval of the posterior distribution for all the model parameters.

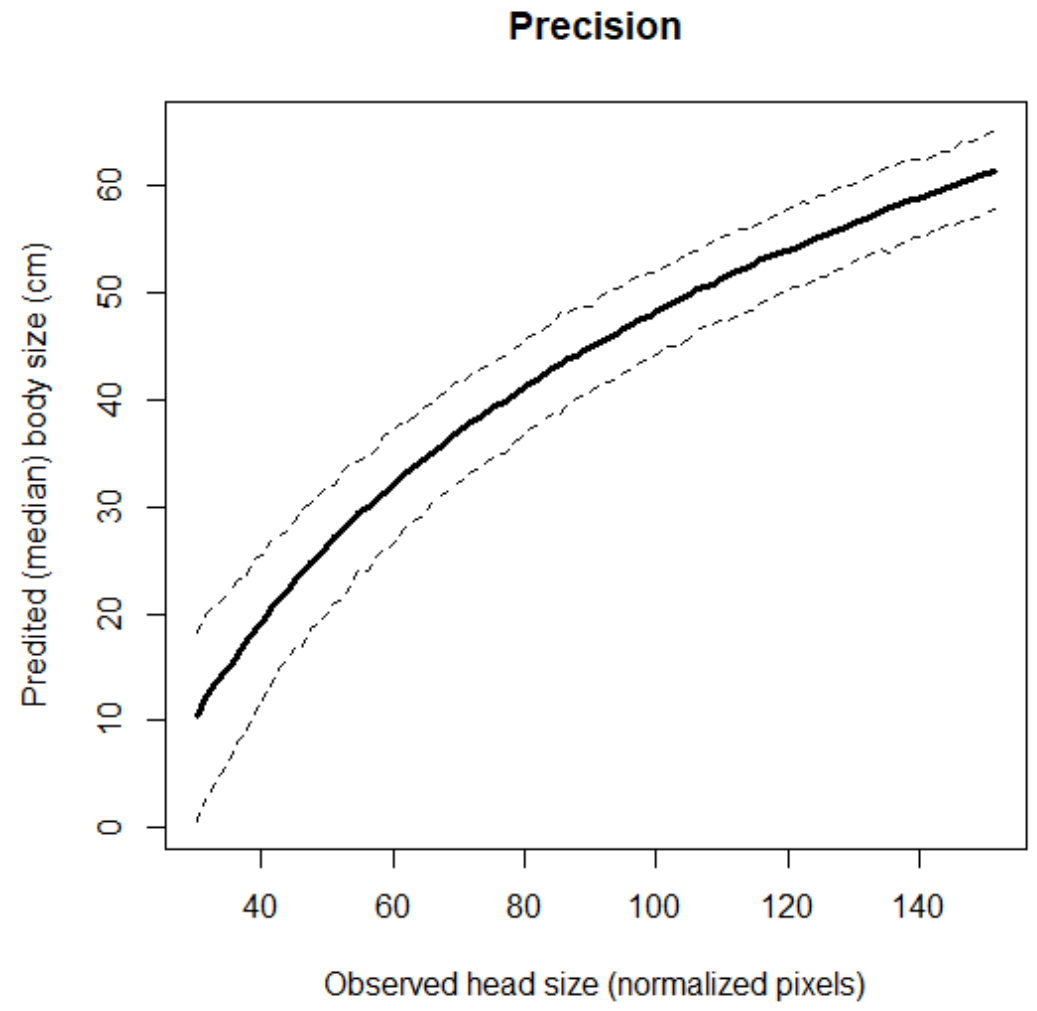

Figure 7: Bootstraped estimates for assessing precision. The median (continuous line) and the 95th quantile range (broken lines) are detailed.

For better assessing the potential sources precision, 100 evenly distributed values along a wide range of HLpix was selected (from 30 to 150 normalized pixels). For each one of those values, 1,000 predictions were estimated. For each one of those 1,000 estimates, a random sample from the posterior distributions of the six model parameters (Table 1) was selected and used to produce an estimate of TL. The 95th quantiles of those 1,000 bootstrapped values are displayed in Figure 7 .

In Table 2, the precision (in terms of $95 \%$ bootstrapped values) are presented for three relevant TL values (i.e., 20, 40 and $60 \mathrm{~cm}$ ). These values are compared with those obtained (using the same bootstrapping procedure) after reducing $50 \%$ each one of the three standard deviations by turn. Note that the three standard deviations are at different scales, thus the method above ensures comparable bootstrap results a properly assessing of the relative importance of the three sources of uncertainty. The 
$20 \mathrm{~cm}$

$40 \mathrm{~cm}$

$60 \mathrm{~cm}$

\begin{tabular}{l|cc|cc|cc} 
& lower & upper & lower & upper & lower & upper \\
\hline Actual & 12.6 & 26.4 & 35.6 & 44.2 & 56.2 & 63.6 \\
$50 \% \sigma_{\text {HLpix }}$ & $\mathbf{1 5 . 3}$ & $\mathbf{2 4 . 4}$ & 36.2 & 43.5 & 56.6 & 63.2 \\
$50 \% \sigma_{\mathrm{HLcm}}$ & 13.3 & 26.5 & 35.4 & 44.4 & 56.4 & 63.6 \\
$50 \% \sigma_{\mathrm{TL}}$ & 13.2 & 25.6 & 36.3 & 43.5 & 57.5 & 62.5
\end{tabular}

Table 2: 95th range of 1,000 bootstrapped estimates of TL from HLpix at three relevant values of TL $(20,40$ and $60 \mathrm{~cm})$. The results from the actually estimated values are compared with those obtained after a $50 \%$ reduction of each one of the three sources of variance considered. Note that this reduction has negligible effects in almost all the cases. The only relevant reduction (denoted in bold) is for small fish when $\sigma_{\text {HLpix }}$ is halved

largest improvement potential is related with $\sigma_{\mathrm{HLpix}}$, which measures the variability between the repeated estimated directly provided by the Deep Learning tool. However, this improvement is clearly restricted to small fish $(20 \mathrm{~cm})$, while the improvement in larger fish is negligible. Note that this uncertainty source pools variability related with posture changes and variability on the object segmentation itself.

\section{Discussion}

In line with many other successful applications of deep learning in a wide range of domains, here we implemented an automatic system for the measurement of fish length from images of captured fish at landing.

The core component of the system is a deep neural network that permits to detect and delineate the contour of the objects of interest, or instances in the deep learning jargon. Here, instead of developing a new network from scratch, a pre-trained Mask R-CNN network was successfully implemented for identifying hake heads. This strategy implies that the network training must be fine tuned with a relatively large data base of examples of hake heads. In this case, the contour of more than 2,000 hake heads have been manually segmented from images, which represent a relevant man-hour investment. However, this strategy seems to well worth if compared to implement a new network from scratch.

The performance of the Mask R-CNN network implemented in this way for detecting hake heads is noteworthy. Almost all the heads in an image are properly detected (99\%) but more interestingly for the specific case of study here, the ratio of false positives is negligible (less than $3 \%$ ). In addition, the confidence score provided by Mask R-CNN may be used for filtering out most of those false positives. Certainly some properly detected heads will be lost after setting an astringent threshold of confidence score for accepting a potential detection, but the number of heads per box and the number of box per day largely ensure that fish length distribution in terms of average size and spread will be properly described.

The specificities of the case study precludes to efficiently detect whole fish contours in the images 
because fish tail are systematically occluded when fisherman prepare the fish boxes. Certainly, other species are not sorted in this careful way but even when the contour of the whole fish is visible on an image, the flexible nature of fish would complicate the performance of the detection step for the Mask R-CNN because the network should be learned with differently bent fish. Conversely, the rigid nature of fish head alleviates this problem but introduces a new handicap because the final objective is not to detect fish heads but estimate fish size. The former output of Mask R-CNN (segmentation mask of the pixels belonging to a given head) was first used to extract head length in pixels. The second step was to transform this head measure form pixels to $\mathrm{cm}$ and the final step was to infer fish length from head length.

Provided that each one of these steps may introduce some uncertainty, a validation protocol has been implemented for assessing the overall performance of the system in terms of accuracy and precision, as well as for providing a reliable confidence interval for fish length estimates. A sample of fish has been measured by an observer with a ruler and these empirical measurements have been compared with the estimates provided by the system developed here. Provided that the median of the measured-estimated differences is virtually zero, and no size-related bias have been detected, the system should be considered accurate.

However, our results showed that there is room for improving the precision of the system. Individual level precision (measured as $95 \%$ quantile) for a newly measured fish length is around $\pm 5 \mathrm{~cm}$. The results of bootstrap simulations suggest that all the variability sources considered are similarly contributing to this suboptimal precision at the fish level. However, uncertainty related with the head posture may be specially relevant for small fish (around $20 \mathrm{~cm}$ ). It is plausible that precise delineation of fish head contour may depend on the fish posture, thus increasing the number of examples of heads in different postures when training the network may alleviate the problem. In addition, the validation protocol should be improved by taking several repeated empirical (i.e., ruler) measurements of each fish and each head because the current model (Equations (1) to (3)) assumes that empirical measures are made without error, thus this potential error source is imputed to the system. Finally, for this specific fish species, the morphometric relationship between head size and fish length has not been particularly good. The anomalies detected for relatively small fish may suggest some ontogenetic change in the allometric relationship between the head and the total size that should be further explored. In any case, enlarging the range of empirically measured fish size and the number of fish sampled are expected to improve precision at the fish level.

Nevertheless, at the current state-of-the-art, we should be prepared for a relatively small precision when compared with the precision attained by an observed with a ruler. Therefore, to implement a validation protocol as the one we proposed here is mandatory for providing not only point estimations for fish length but also reliable confidence intervals. In this sense, the hierarchical Bayesian framework 
proposed here is not only appropriate for providing reliable confidence intervals at fish level. Moreover, it can be expanded for properly propagate such a fish level uncertainly to the fish box level, the boat level, the day level or any other relevant scale that might be of interest in other cases study. Specifically, in the context of understanding fish population dynamics and to take informed management decisions on exploited stocks, a relatively low precision at the fish level may be largely compensated with a massive amount of data at upper scales.

It is in this context that the advancement in marine science is foreseen to be boosted in the next few years thanks to the capacity of generating massive amounts of data from automatic sensors coupled to high-power computation capabilities (Lowerre-Barbieri et al., 2019; Danovaro et al., 2017). Many techniques associated to the Artificial intelligence are not new in marine science (e.g. simple neural networks, decision trees or Bayesian networks). Many of these techniques are used for ecosystem modeling purposes, spatial planning, decision making etc (e.g. (Fernandes et al., 2010)). However, the field of Deep Learning is advancing at a greater pace, an in particular those applications related with image processing.

Until now, most applications of image classification in marine ecology were semi-supervised or supervised (e.g. (Marini et al., 2016), (Marini et al., 2018), (Díaz-Gil et al., 2017)). Through Deep Learning we exploit the structural characteristics of data and make use of Big Data computation capabilities (Hu et al., 2014), which, in our case study, may revolutionize more conventional ways of extracting data. We clearly demonstrate that by using this method, even when only a percentage of the fish in each box can be correctly assigned to a size, opens the opportunity to massive fish length sampling of many commercially valuable species, without interference with wharf or fishing operations and activity. Provided that an image of each fish box can be easily obtained and stored when the conveyor belt stops for bidding, the estimate number of pictures (each box) per day that currently are arriving to our system are in the order of thousands. This knowledge will enable to improve not only the current biological evaluation models based in size, but also to explore short-term effects of the environment on the species, or even the analysis of price dynamics within the season in relation to size. To this end, we have detected a very positive attitude from the fishery sector, which suggests that the development of these techniques in the near future are guaranteed. According to the above, several near-future improvements are envisaged, including i) the detection of different species in the same image (some boxes contain a mixture of species), ii) the automatic calibration of cameras for the conversion from pixel unit lengths to centimeters and iii) an improvement of the better estimation of total fish lengths from pelvic lengths.

\section{Acknowledgements}

This work has been funded by the projects FOTOPEIX and FOTOPEX2 (2017/2279, 2018/2002) from Fundación Biodiversidad, through the Pleamar Program. We specially thank OPMALLORCAMAR 
and Direcció General de Pesca del Govern de les Illes Balears for supporting these projects. The work of J.L.Lisani was partially supported by grants TIN2017-85572-P and DPI2017-86372-C3-3-R (MINECO/AEI/FEDERUE). This is a contribution of the Unitat Associada IMEDEA-LIMIA.

\section{References}

Abdullah, N., M. Shafry, M. Rahim, and I. M. Amin

2009. Measuring fish length from digital images (filedi). In Proceedings of the $2 N d$ International Conference on Interaction Sciences: Information Technology, Culture and Human, Pp. 38-43. ACM.

Al-Jubouri, Q., W. Al-Nuaimy, M. Al-Taee, and I. Young

2017. An automated vision system for measurement of zebrafish length using low-cost orthogonal web cameras. Aquacultural Engineering, 78:155 - 162.

Arnarson, H., K. Bengoetxea, and L. Pau

1991. Vision applications in the fishing and fish product industries. Fish Quality Control by Computer Vision, Pp. 21-41.

Chen, G., P. Sun, and Y. Shang

2017. Automatic fish classification system using deep learning. In 2017 IEEE 29th International Conference on Tools with Artificial Intelligence (ICTAI), Pp. 24-29.

Costa, C., A. Loy, S. Cataudella, D. Davis, and M. Scardi

2006. Extracting fish size using dual underwater cameras. Aquacultural Engineering, 35(3):218 - 227.

Dalal, N. and B. Triggs

2005. Histograms of oriented gradients for human detection. In 2005 IEEE Computer Society Conference on Computer Vision and Pattern Recognition (CVPR'05), volume 1, Pp. 886-893 vol. 1.

Danovaro, R., J. Aguzzi, E. Fanelli, D. Billett, K. Gjerde, A. Jamieson, E. Ramirez-Llodra, C. R. Smith, P. V. R. Snelgrove, L. Thomsen, and C. L. V. Dover 2017. An ecosystem-based deep-ocean strategy. Science, 355(6324):452-454.

Díaz-Gil, C., S. L. Smee, L. Cotgrove, G. Follana-Berná, H. Hinz, P. Marti-Puig, A. Grau, M. Palmer, and I. A. Catalán

2017. Using stereoscopic video cameras to evaluate seagrass meadows nursery function in the mediterranean. 164(6):137. Exported from https://app.dimensions.ai on 2019/05/03.

d'Armengol, L., M. P. Castillo, I. Ruiz-Mallén, and E. Corbera

2018. A systematic review of co-managed small-scale fisheries: Social diversity and adaptive management improve outcomes. Global Environmental Change, 52:212 - 225 . 
FAO

2016. The state of Mediterranean and Black sea Fisheries. Technical report, FAO.

FAO

2018. The State of World Fisheries and Aquaculture (SOFIA). Technical report, FAO.

Fernandes, J. A., X. Irigoien, N. Goikoetxea, J. A. Lozano, I. Inza, A. Pérez, and A. Bode

2010. Fish recruitment prediction, using robust supervised classification methods. Ecological Modelling, $221(2): 338-352$.

Gelman, A., J. B. Carlin, H. S. Stern, and D. B. Rubin

2003. Bayesian Data Analysis, volume 45.

Hardin, R. W.

2006. Vision system monitors fish populations. Vision Systems Design, 11(1):41,43-45.

He, K., G. Gkioxari, P. Dollár, and R. Girshick

2017. Mask r-cnn. In 2017 IEEE International Conference on Computer Vision (ICCV), Pp. 29802988.

Hilborn, R.

2007. Managing fisheries is managing people: what has been learned? Fish and Fisheries, 8:285-296.

Hu, H., Y. Wen, T. Chua, and X. Li

2014. Toward scalable systems for big data analytics: A technology tutorial. IEEE Access, 2:652-687.

Iudicello, S., M. L. Weber, and R. Wieland

2012. Fish, Markets, and Fishermen: The Economics Of Overfishing. Island Press.

Krizhevsky, A., I. Sutskever, and G. E. Hinton

2012. Imagenet classification with deep convolutional neural networks. In Proceedings of the 25th International Conference on Neural Information Processing Systems - Volume 1, NIPS'12, Pp. 10971105, USA. Curran Associates Inc.

Lecun, Y., L. Bottou, Y. Bengio, and P. Haffner

1998. Gradient-based learning applied to document recognition. Proceedings of the IEEE, 86(11):22782324 .

Lin, T., P. Dollár, R. Girshick, K. He, B. Hariharan, and S. Belongie

2017. Feature pyramid networks for object detection. In 2017 IEEE Conference on Computer Vision and Pattern Recognition (CVPR), Pp. 936-944. 
Lowerre-Barbieri, S. K., I. A. Catalán, A. Frugård Opdal, and C. Jørgensen

2019. Preparing for the future: integrating spatial ecology into ecosystem-based management. ICES Journal of Marine Science, 76(2):467-476.

Marini, S., E. Azzurro, S. Coco, J. del Rio, E. S, E. Fanelli, M. Nogueras, V. Sbragaglia, D. Toma, and J. Aguzzi

2016. Automatic fish counting from underwater video images: performance estimation and evaluation. In 7th International Workshop on Marine Technology.

Marini, S., E. Fanelli, V. Sbragaglia, E. Azzurro, J. Del Rio Fernandez, and J. Aguzzi 2018. Tracking fish abundance by underwater image recognition. Sci. Rep., 8:13748.

Martinez-de Dios, J. R., C. Serna, and A. Ollero

2003. Computer vision and robotics techniques in fish farms. Robotica, 21(3):233-243.

Maynou, F., B. Morales-Nin, M. Cabanellas-Reboredo, M. Palmer, E. García, and A. Grau

2013. Small-scale fishery in the balearic islands (w mediterranean): A socio-economic approach. Fisheries Research, 139:11-17.

Meng, L., T. Hirayama, and S. Oyanagi

2018. Underwater-drone with panoramic camera for automatic fish recognition based on deep learning. IEEE Access, 6:17880-17886.

Miranda, J. M. and M. Romero

2017. A prototype to measure rainbow trout's length using image processing. Aquacultural Engineering, $76: 41-49$.

Morales-Nin, B., A. Grau, and M. Palmer

2010. Managing coastal zone fisheries: A mediterranean case study. Ocean $\&$ Coastal Management, $53: 99-106$.

Palmer, M., A. Quetglas, B. Guijarro, J. Moranta, F. Ordines, and E. Massutí

2009. Performance of artificial neural networks and discriminant analysis in predicting fishing tactics from multispecific fisheries. Canadian Journal of Fisheries and Aquatic Sciences, 66:224-237.

Palmer, M., B. Tolosa, A. Grau, C. del Mar Gil, M.and Obregón, and B. Morales-Nin

2017. Combining sale records of landings and fishers knowledge for predicting métiers in a small-scale, multi-gear, multispecies fishery. Fisheries research, 195:59-70.

Petrell, R., X. Shi, R. Ward, A. Naiberg, and C. Savage 1997. Determining fish size and swimming speed in cages and tanks using simple video techniques. Aquacultural Engineering, 16(1):63 - 84 . 
Plummer, M., N. Best, K. Cowles, and K. Vines

2006. Coda: convergence diagnosis and output analysis for mcmc. $R$ News, 6(1):7-11.

Qin, H., X. Li, J. Liang, Y. Peng, and C. Zhang

2016. Deepfish: Accurate underwater live fish recognition with a deep architecture. Neurocomputing, 187:49 - 58. Recent Developments on Deep Big Vision.

Redmon, J., S. Divvala, R. Girshick, and A. Farhadi

2016. You only look once: Unified, real-time object detection. Pp. 779-788.

Reglero, P. and B. Morales-Nin

2008. Relationship between first sale price, body size and total catch of trammelnet target species in majorca (nw mediterranean). Fisheries Research - FISH RES, 92:102-106.

Ren, S., K. He, R. Girshick, and J. Sun

2015. Faster r-cnn: Towards real-time object detection with region proposal networks. IEEE Transactions on Pattern Analysis and Machine Intelligence, 39.

Strachan, N.

1993. Length measurement of fish by computer vision. Computers and Electronics in Agriculture, $8(2): 93-104$.

Tillett, R., N. McFarlane, and J. Lines

2000. Estimating dimensions of free-swimming fish using $3 \mathrm{~d}$ point distribution models. Computer Vision and Image Understanding, 79(1):123 - 141.

Viola, P. and M. J. Jones

2004. Robust real-time face detection. Int. J. Comput. Vision, 57(2):137-154.

White, D., C. Svellingen, and N. Strachan

2006. Automated measurement of species and length of fish by computer vision. Fisheries Research, $80(2): 203-210$.

Zion, B., V. Alchanatis, V. Ostrovsky, A. Barki, and I. Karplus 2007. Real-time underwater sorting of edible fish species. Computers and Electronics in Agriculture, $56(1): 34-45$. 